PROCEEDINGS OF THE

AMERICAN MATHEMATICAL SOCIETY

Volume 134, Number 3, Pages 755-763

S 0002-9939(05)08025-1

Article electronically published on July 19, 2005

\title{
FUNCTIONAL CALCULUS AND *-REGULARITY OF A CLASS OF BANACH ALGEBRAS
}

\author{
CHI-WAI LEUNG AND CHI-KEUNG NG
}

(Communicated by David R. Larson)

\begin{abstract}
Suppose that $(A, G, \alpha)$ is a $C^{*}$-dynamical system such that $G$ is of polynomial growth. If $A$ is finite dimensional, we show that any element in $K(G ; A)$ has slow growth and that $L^{1}(G, A)$ is $*$-regular. Furthermore, if $G$ is discrete and $\pi$ is a "nice representation" of $A$, we define a new Banach *-algebra $l_{\pi}^{1}(G, A)$ which coincides with $l^{1}(G ; A)$ when $A$ is finite dimensional. We also show that any element in $K(G ; A)$ has slow growth and $l_{\pi}^{1}(G, A)$ is *-regular.
\end{abstract}

\section{INTRODUCTION AND PRELIMINARIES}

For a Banach $*$-algebra $B$, we denote by $B^{\sim}$ the unitalization of $B$ together with the $*$-algebra norm defined by $\|b+\lambda 1\|=\|b\|+|\lambda|$. We also denote by $C^{*}(B)$ the enveloping $C^{*}$-algebra of $B$ and $\Phi: B \rightarrow C^{*}(B)$ the canonical embedding (not necessarily injective).

In the following, we assume that $C^{*}(B) \neq(0)$. Moreover, throughout this paper, all *-representations of Banach $*$-algebras are assumed to be non-degenerate and all ideals are closed. For any $*$-representation $\pi$ of $B$, there is a unique $*$-representation $\pi_{*}$ of $C^{*}(B)$ such that $\pi=\pi_{*} \circ \Phi$. Let Prim $C^{*}(B)$ be the space of primitive ideals of $C^{*}(B)$ and let $\operatorname{Prim}_{*} B$ be the space of kernels of topological irreducible $*$-representations of $B$, both equipped with the Jacobson topology. Moreover, $\Phi$ induces a continuous surjection $\Psi: \operatorname{Prim} C^{*}(B) \rightarrow \operatorname{Prim}_{*} B$ (see e.g. 7, Corollary 10.5.7]).

Definition $1.1([7,10.5 .8])$. A Banach $*$-algebra $B$ is said to be $*$-regular if the canonical map $\Psi: \operatorname{Prim} C^{*}(B) \rightarrow \operatorname{Prim}_{*} B$ is a homeomorphism.

Remark 1.2. $B$ is $*$-regular if and only if $B / *-\operatorname{rad}(B)$ is $*$-regular $($ where $*-\operatorname{rad}(B)$ is the $*$-radical of $B$ ).

We now recall the following result from [2]. As we are in a more general setting, we repeat their argument here for clarity.

Received by the editors June 23, 2004 and, in revised form, August 19, 2004 and October 13, 2004 .

2000 Mathematics Subject Classification. Primary 47A60, 32A65.

Key words and phrases. Banach algebras, functional calculus, *-regular.

This work was jointly supported by Hong Kong RGC Direct Grant and the National Natural Science Foundation of China (10371058). 
Proposition 1.3 ([2, Satz 1]). A Banach *-algebra B is *-regular if and only if for any $*$-representations $\pi$ and $\rho$ of $B$, the inclusion $\operatorname{ker} \pi \subseteq \operatorname{ker} \rho$ will imply that $\|\rho(b)\| \leq\|\pi(b)\|$ for all $b \in B$.

Proof. Suppose that $B$ is $*$-regular. Let

$$
E:=\left\{P \in \operatorname{Prim} C^{*}(B): \operatorname{ker} \pi_{*} \subseteq P\right\} .
$$

As $\Phi(\operatorname{ker} \pi)=\operatorname{ker} \pi_{*} \cap \Phi(B)$, we see that $\bigcap_{P \in E} \Phi(\Psi(P))=\bigcap_{P \in E} P \cap \Phi(B)=$ $\Phi(\operatorname{ker} \pi)$. Let $I \in \operatorname{Prim} C^{*}(B)$ such that $\operatorname{ker} \rho_{*} \subseteq I$. Then

$$
\bigcap_{P \in E} \Phi(\Psi(P)) \subseteq \Phi(\operatorname{ker} \rho) \subseteq I \cap \Phi(B)=\Phi(\Psi(I)),
$$

which implies that $\bigcap_{Q \in \Psi(E)} Q \subseteq \Psi(I)$ (as $\operatorname{ker} \Phi \subseteq J$ for any $J \in \operatorname{Prim}_{*} B$ ). Since $\Psi$ is a homeomorphism and $E$ is closed, $\Psi(I) \in \Psi(E)$ and $I \in E$. This shows that $\operatorname{ker} \pi_{*} \subseteq \operatorname{ker} \rho_{*}$ and so $\left\|\rho_{*}(x)\right\| \leq\left\|\pi_{*}(x)\right\|$ for any $x \in C^{*}(B)$. Conversely, the hypothesis clearly implies that $\Psi$ is injective (as $\operatorname{ker} \pi=\operatorname{ker} \rho$ will then imply $\left\|\rho_{*}(x)\right\|=\left\|\pi_{*}(x)\right\|$ for any $\left.x \in C^{*}(B)\right)$. Let $E \subseteq$ Prim $C^{*}(B)$ be a closed subset. For any $\operatorname{ker} \tau \in \operatorname{hull}(\operatorname{ker} \Psi(E))$, we have

$$
\operatorname{ker} \tau \supseteq \bigcap_{\operatorname{ker} \sigma_{*} \in E} \operatorname{ker} \sigma=\operatorname{ker}\left(\bigoplus_{\operatorname{ker} \sigma_{*} \in E} \sigma\right) .
$$

Therefore, by the hypothesis, $\left\|\tau_{*}(x)\right\| \leq \sup \left\{\left\|\sigma_{*}(x)\right\|: \operatorname{ker} \sigma_{*} \in E\right\} \quad\left(x \in C^{*}(B)\right)$. Hence $\operatorname{ker} \tau_{*} \in E$ (as $E$ is closed) and $\operatorname{ker} \tau \in \Psi(E)$.

Corollary 1.4. Let $B$ be a Banach *-algebra.

(a) Suppose that there is a dense subset $B_{0}$ of $B_{s a}$ such that for any $b \in B_{0}$ and any smooth function $\varphi: \mathbb{R}_{+} \rightarrow \mathbb{C}$ with compact support and $\varphi(0)=0$, there exists $c \in B$ with $\mu(c)=\varphi\left(\mu\left(b^{2}\right)\right)$ for any $*$-representation $\mu$ of $B$. Then $B$ is $*$-regular.

(b) Suppose that $\left\{B_{i}\right\}_{i \in I}$ is a directed family of Banach $*$-subalgebras of $B$ (i.e. $B_{i} \subseteq B_{j}$ if $\left.i \leq j\right)$ such that $\bigcup_{i \in I} B_{i}$ is dense in $B$. If all $B_{i}$ are $*$-regular, then so is $B$.

Proof. (a) Let $\left(\pi, H_{\pi}\right)$ and $\left(\rho, H_{\rho}\right)$ be two $*$-representations of $B$ such that ker $\pi \subseteq$ ker $\rho$. Assume that there exists $a \in B$ such that $\|\pi(a)\|<\|\rho(a)\|$. By the density of $B_{0}$ and the $C^{*}$-identity, there exists $b \in B_{0}$ such that $\left\|\pi\left(b^{2}\right)\right\|<\left\|\rho\left(b^{2}\right)\right\|$. Consider a smooth function $\varphi: \mathbb{R}_{+} \rightarrow \mathbb{C}$ with compact support such that

$$
\varphi\left(\mathbb{R}_{+}\right) \subseteq[0,1], \quad \varphi\left(\left[0,\left\|\pi\left(b^{2}\right)\right\|\right]\right)=\{0\} \quad \text { and } \quad \varphi\left(\left\|\rho\left(b^{2}\right)\right\|\right)=1 .
$$

Let $c \in B$ be the element given by the hypothesis. Then $\pi(c)=\varphi\left(\pi\left(b^{2}\right)\right) \in \mathcal{L}\left(H_{\pi}\right)_{+}$. From the equalities:

$$
\sigma(\pi(c))=\varphi\left(\sigma\left(\pi\left(b^{2}\right)\right)\right)=\{0\},
$$

we see that $c \in \operatorname{ker} \pi$, where $\sigma(x)$ denotes the spectrum of $x$. However, $\sigma(\rho(c))=$ $\varphi\left(\sigma\left(\rho\left(b^{2}\right)\right)\right) \neq\{0\}$, which gives the contradiction that $c \notin \operatorname{ker} \rho$.

(b) Suppose that $\pi$ and $\rho$ are two $*$-representations of $B$ such that $\operatorname{ker} \pi \subseteq \operatorname{ker} \rho$. It is clear that for any $i \in I$, one has

$$
\operatorname{ker}\left(\left.\pi\right|_{B_{i}}\right) \subseteq \operatorname{ker}\left(\left.\rho\right|_{B_{i}}\right)
$$

(where $\left.\pi\right|_{B_{i}}$ is the non-degenerated part of the restriction of $\pi$ on $B_{i}$ and so is $\left.\rho \mid B_{i}\right)$. Therefore, by Proposition 1.3. $\|\rho(b)\| \leq\|\pi(b)\|\left(b \in B_{i}\right)$. Now, the result follows from the density of $\bigcup_{i \in I} B_{i}$ as well as Proposition 1.3 . 
Remark 1.5. (a) Corollary 1.4(a) is the argument in [2, Satz 2]. Note that we do not assume that the spectrum of $b^{2}$ is in $\mathbb{R}_{+}$.

(b) Recall that $b \in B$ is said to have slow growth if there exists $k \in \mathbb{N}$ such that $\left\|e^{i t b}\right\|=O\left(|t|^{k}\right)$ for $t \in \mathbb{R}$ (see [1]).

It was proved in 22 that $L^{1}(G)$ is a *-regular Banach $*$-algebra if $G$ is a polynomial growth group. The main step in their proof is the observation that if $G$ is of polynomial growth, then by the argument of a main result in [3], any self-adjoint element in $K(G)$ (the space of continuous functions with compact supports) has slow growth. It is natural to ask if a similar thing holds for a $C^{*}$-dynamical system $(A, G, \alpha)$. In this paper, we will consider two particular cases: the case when $A$ is finite dimensional and the case when $G$ is discrete.

Notation 1.6. Throughout this paper, $(A, G, \alpha)$ is a $C^{*}$-dynamical system. For any $f, g \in K(G ; A)$ (continuous maps from $G$ to $A$ with compact supports) and $s \in G$, we define

$$
(f \star g)(s):=\int_{G} \alpha_{t}(f(s t)) g\left(t^{-1}\right) d t \quad \text { and } \quad f^{\#}(s):=\Delta\left(s^{-1}\right) \alpha_{s^{-1}}\left(f\left(s^{-1}\right)^{*}\right)
$$

(see [5, 2.3]).

If $\Theta$ is a map from $K(G ; A)$ to itself defined by $\Theta(f)(t)=\alpha_{t}^{-1}(f(t))$, then we have $\Theta(f * g)=\Theta(f) \star \Theta(g)$ and $\Theta\left(f^{*}\right)=\Theta(f)^{\#}$ (where $f * g(s):=\int_{G} f(r) \alpha_{r}\left(g\left(r^{-1} s\right)\right) d r$ and $f^{*}(t):=\Delta(t)^{-1} \alpha_{t}\left(f\left(t^{-1}\right)^{*}\right)$. Moreover, any covariant representation $(\pi, u)$ of $(A, G, \alpha)$, i.e.

$$
\pi\left(\alpha_{r}(a)\right)=u_{r} \pi(a) u_{r-1}
$$

induces a $*$-representation $u \star \pi$ of $(K(G, A), \star, \#)$ which is defined by

$$
u \star \pi(f) \xi=\int_{G} u_{t} \pi(f(t)) \xi d t .
$$

\section{The finite Dimensional $C^{*}$-Algebra CASE}

Throughout this section, we assume that in the $C^{*}$-dynamical system $(A, G, \alpha)$, $A$ is a finite dimensional $C^{*}$-algebra with the $C^{*}$-norm $\|\cdot\|_{A}$. Suppose that $A=$ $\bigoplus_{k=1}^{n} M_{m_{k}}$. Then $\operatorname{Tr}:=\frac{1}{n} \sum_{k=1}^{n} \operatorname{Tr}_{m_{k}}$ is a normalised trace on $A$ (where $\operatorname{Tr}_{m_{k}}$ is the normalised trace on the component $M_{m_{k}}$ ). Since any automorphism of $A$ is the composition of an inner automorphism with a swapping of components of $A$ that have the same dimensions (i.e. $m_{k}$ ), $\operatorname{Tr}$ is $\alpha$-invariant (i.e. $\operatorname{Tr}\left(\alpha_{s}(a)\right)=\operatorname{Tr}(a)$ for any $a \in A$ and $s \in G)$.

Remark 2.1. (a) Suppose that $\left(H_{T}, \rho\right)$ is the GNS representation corresponding to Tr. Let $\mathcal{H}$ be the Hilbert space $L^{2}(G) \otimes H_{T}$. If we regard $A \subseteq H_{T}$ and $K(G ; A) \subseteq$ $L^{2}(G) \otimes H_{T}$, then for any $f, g \in K(G ; A)$, we have

$$
(f, g)_{\mathcal{H}}=\int_{G} \operatorname{Tr}\left(f(t)^{*} g(t)\right) d t
$$

(note that we use the convention that the inner product is anti-linear in the first variable).

(b) Let $f$ be a measurable map from $G$ to $A$ (i.e., there exists a sequence of measurable simple maps that converges to $f$ almost everywhere). As usual, we 
define, for $1 \leq p<\infty$,

and

$$
\begin{aligned}
\|f\|_{p} & :=\left(\int_{G}\|f(t)\|_{A}^{p} d t\right)^{1 / p} \\
\|f\|_{\infty} & :=\inf \left\{\sup _{t \in \Delta}\|f(t)\|_{A}: \Delta \subseteq G ; \mu_{G}(G \backslash \Delta)=0\right\}
\end{aligned}
$$

where $\mu_{G}$ is the Haar measure on $G$. Let $L^{p}(G ; A)=\{f: G \rightarrow A \mid f$ is measurable and $\left.\|f\|_{p}<\infty\right\}$ (strictly speaking, we identify two such maps if they coincide almost everywhere). It is well known that $\left(L^{1}(G ; A), \star, \#\right)$ is a Banach $*$-algebra under this norm. Moreover, since $A$ is finite dimensional, $\|\cdot\|_{2}$ is equivalent to the norm $\|\cdot\|_{\mathcal{H}}$ on $K(G ; A)$ and so, $L^{2}(G ; A) \cong \mathcal{H}$ (as Banach spaces).

(c) Consider the Hilbert $A$-module $L^{2}(G) \otimes A$ with the $A$-inner product

$$
\langle\phi \otimes a, \psi \otimes b\rangle_{A}:=\left(\int_{G} \phi(t)^{*} \psi(t) d t\right) a^{*} b
$$

$\left(\phi, \psi \in L^{2}(G) ; a, b \in A\right)$. Then the canonical $*$-homomorphism $\mu: L^{1}(G ; A) \rightarrow$ $\mathcal{L}_{A}\left(L^{2}(G) \otimes A\right)$ induces an injective $*$-representation $T: L^{1}(G, A) \rightarrow \mathcal{L}(\mathcal{H})$ (note that $\left.\mathcal{H}=\left(L^{2}(G) \otimes A\right) \otimes_{\rho} H_{T}\right)$.

Lemma 2.2. (a) If $f \in K(G ; A)$, then $\sum_{k=1}^{\infty} \frac{(i f)^{k}}{k !}$ converges to $u(f)$ in $L^{1}(G ; A) \cap$ $C_{0}(G ; A)$ (and so we can regard $u(f)$ as an element in $L^{2}(G ; A) \cong \mathcal{H}$ ).

(b) Suppose that $G$ is unimodular. If $g, h \in L^{1}(G ; A) \cap L^{\infty}(G ; A)$ such that $T(g)^{*} T(g) \leq T(h)^{*} T(h)$, then $\|g\|_{\mathcal{H}} \leq\|h\|_{\mathcal{H}}$.

Proof. (a) For any $k, l \in K(G ; A)$, we have $\|k \star l\|_{\infty} \leq\|k\|_{1}\|l\|_{\infty}$. Therefore,

$$
\sum_{n=1}^{\infty} \frac{\left\|(i f)^{n}\right\|_{\infty}}{n !} \leq \sum_{n=0}^{\infty} \frac{\|f\|_{1}^{n}}{(n+1) !}\|f\|_{\infty} \leq e^{\|f\|_{1}}\|f\|_{\infty}<\infty
$$

and $u(f) \in C_{0}(G ; A)$.

(b) By the assumption, for all $\xi \in K(G ; A) \subseteq \mathcal{H}$,

$$
\|g \star \xi\|_{\mathcal{H}}^{2}=\left(T(g)^{*} T(g) \xi, \xi\right)_{\mathcal{H}} \leq\left(T(h)^{*} T(h) \xi, \xi\right)_{\mathcal{H}}=\|h \star \xi\|_{\mathcal{H}}^{2} .
$$

If $\left(f_{j}\right)_{j \in I}$ is a net in $L^{1}(G ; A) \cap L^{\infty}(G ; A)$ such that $\left\|f_{j}\right\|_{1} \rightarrow 0$ and there exists $\kappa \in \mathbb{R}_{+}$with $\left\|f_{j}\right\|_{\infty}<\kappa$ (for all $j \in I$ ), then

$$
\left\|f_{j}\right\|_{\mathcal{H}}^{2}=\int_{G} \operatorname{Tr}\left[f_{j}(t)^{*} f_{j}(t)\right] d t \leq \kappa\|\operatorname{Tr}\| \int_{G}\left\|f_{j}(t)\right\|_{A} d t .
$$

Now suppose that $\left(\xi_{i}\right) \subseteq K(G ; A)$ is a contractive approximate identity for $L^{1}(G ; A)$. Notice that as $G$ is unimodular, $\|k \star l\|_{\infty} \leq\|k\|_{\infty}\|l\|_{1}$ for any $k, l \in$ $K(G ; A)$. Thus $\left\|g \star \xi_{i}-g\right\|_{\infty} \leq 2\|g\|_{\infty}$ and $\left\|g \star \xi_{i}-g\right\|_{1} \rightarrow 0$. Therefore, inequality (2.2) implies that $\left\|g \star \xi_{i}-g\right\|_{\mathcal{H}} \rightarrow 0$ and the same is true for $h$. Now, the required inequality follows from (2.1).

Proposition 2.3. Suppose that $G$ is of polynomial growth and $f \in K(G ; A)$ with $f^{\#}=f$. Then $f$ has slow growth.

Proof. By Lemma 2.2, [3, Lemme 4] and Remark 2.1 (b), we see that $\|u(f)\|_{2} \leq$ $C_{0}\|f\|_{2}$ for some constant $C_{0}>0$. Now, the same argument as that in [3, Lemme $6]$ will imply the result. 
Using the above proposition and the argument in [3, Lemme 7], the "smooth functional calculus" can be defined for any $f \in K(G ; A)_{s a}$ in such a way that the hypothesis of Corollary 1.4(a) holds. Since $K(G ; A)_{s a}$ is dense in $L^{1}(G ; A)_{s a}$, we see that $L^{1}(G ; A)$ is *-regular. This gives the following generalisations of [2, Satz 2]. Note that part (c) is also a partial generalisation of [6, Remark 1] (i.e. $L^{1}(G ; A)$ is *-regular if $G$ is abelian).

Theorem 2.4. Suppose that $G$ is a polynomial growth group, $A$ is a finite dimensional $C^{*}$-algebra and $\alpha$ is an action of $G$ on $A$. Let $f \in K(G ; A)$ with $f=f^{\#}$ and let $\varphi$ be a smooth and integrable complex function on $\mathbb{R}$.

(a) $\varphi\{f\}:=\frac{1}{2 \pi} \int_{-\infty}^{\infty} \hat{\varphi}(r) e^{i r f} d r$ exists in the unitalisation, $L^{1}(G ; A)^{\sim}$, of $L^{1}(G ; A)$ (where $\hat{\varphi}$ is the Fourier transform of $\varphi$ ) and $\varphi\{f\} \in L^{1}(G ; A)$ if $\varphi(0)=0$.

(b) For any covariant representation $(\nu, v)$ of $(A, G, \alpha)$, we have $v \star \nu(\varphi\{f\})=$ $\varphi(v \star \nu(f))$.

(c) $L^{1}(G ; A)$ is *-regular.

\section{The Discrete Group CASE}

In this section, we will consider the case when $G$ is a discrete group (but $A$ is a general $C^{*}$-algebra). The absence of a bounded trace that gives an equivalent norm on $A$ makes the situation much more complicated.

Let us start with the easy case when $G$ is a "locally finite group". We recall the well-known fact that if $G$ is finite, then $K(G ; A)=l^{1}(G ; A)=A \times{ }_{\alpha} G$ (note that if $\left\{f_{n}\right\}$ is a sequence in $K(G ; A)$ converging to an element in $A \times_{\alpha} G$, then $\left\{f_{n}(t)\right\}$ is Cauchy for any $t \in G$ and so $\left\{f_{n}\right\}$ converges in $\left.K(G ; A)\right)$.

Proposition 3.1. Let $G$ be the inductive limit of a system of finite groups $\left\{G_{i}\right\}_{i \in I}$ and let $\alpha$ be an action of $G$ on a $C^{*}$-algebra $A$. Then for any $f \in K(G ; A)$ with $f=f^{\#}$ and any smooth and integrable complex function $\varphi$ on $\mathbb{R}, \varphi\{f\}:=$ $\frac{1}{2 \pi} \int_{-\infty}^{\infty} \hat{\varphi}(r) e^{i r f} d r$ exists in the $K(G ; A)^{\sim}$ and $l^{1}(G ; A)$ is *-regular.

Proof. Note that $K\left(G_{i} ; A\right)=A \times \alpha_{\alpha_{i}} G_{i}$ is *-regular $\left(\alpha_{i}\right.$ being the restriction of $\alpha$ on $\left.G_{i}\right)$. Moreover, it is easy to see that $\bigcup_{i \in I} l^{1}\left(G_{i}\right)$ is dense in $l^{1}(G)$. Since $l^{1}(G ; A)=l^{1}(G) \otimes^{\pi} A$ (where $\otimes^{\pi}$ is the projective tensor product), it is easy to see that $\bigcup_{i \in I} l^{1}\left(G_{i} ; A\right)$ is dense in $l^{1}(G ; A)$. Now this result follows from Corollary 1.4(b). The existence of the functional calculus follows from the fact that for any $f \in K(G ; A)$ there exists $i \in I$ such that $f \in K\left(G_{i} ; A\right)=A \times_{\alpha_{i}} G_{i}$.

Next, we consider the case when $G$ is discrete and has polynomial growth. As in the previous section, we want to use a similar argument to that of [3]. In order to do this, we need to replace $\left\{\|\cdot\|_{p}\right\}_{p \in[1, \infty]}$ by another series of norms $\left\{n_{\pi, p}\right\}_{p \in[1, \infty]}$ such that $n_{\pi, 2}$ is the one given by the Hilbert $C^{*}$-module $l^{2}(G) \otimes A$.

Remark 3.2. Suppose that $\pi$ is a representation of $A$ on $H$. For any $f: G \rightarrow A$ and any $1 \leq p<\infty$, we define

$$
n_{\pi, p}(f)=\sup \left\{\left(\sum_{t \in G}\|\pi(f(t)) \xi\|^{p}\right)^{1 / p}: \xi \in H \text { and }\|\xi\| \leq 1\right\} .
$$

(a) It is clear that $n_{\pi, p}$ is a semi-norm on $K(G ; A)$ and if $\pi$ is faithful, then $n_{\pi, p}$ is a norm on $K(G ; A)$. Moreover, $n_{\pi, p}(f) \leq\|f\|_{p}$. 
(b) Suppose that $f \in K(G ; A)$. Then

$$
\sum_{t \in G}\|\pi(f(t)) \xi\|^{2}=\left\langle\pi\left(\sum_{t \in G} f(t)^{*} f(t)\right) \xi, \xi\right\rangle .
$$

Hence if $\pi$ is faithful, then $n_{\pi, 2}^{2}(f)=\left\|\sum_{t \in G} f(t)^{*} f(t)\right\|$ and $n_{\pi, 2}$ is the norm on $K(G ; A)$ induced from the Hilbert $A$-module $l^{2}(G) \otimes A$.

(c) Suppose that $\xi \in H$ and $f, g \in K(G ; A)$. Then

$$
\begin{aligned}
\sum_{s \in G}\|\pi(f \star g(s)) \xi\| & \leq \sum_{v \in G} \sum_{u \in G}\left\|\pi\left(\alpha_{v^{-1}}(f(u)) g(v)\right) \xi\right\| \\
& \leq \sum_{v \in G} \sum_{u \in G}\|f(u)\|\|\pi(g(v)) \xi\| \leq\|f\|_{1} n_{\pi, 1}(g)\|\xi\|
\end{aligned}
$$

and so, $n_{\pi, 1}(f \star g) \leq\|f\|_{1} n_{\pi, 1}(g)$.

(d) In general, it may not be true that $n_{\pi, p}(f)=n_{\pi, p}\left(f^{\#}\right)$.

Proposition 3.3. Suppose that $A$ is finite dimensional and $\pi$ is any faithful representation of $A$. Then $n_{\pi, 1} \sim\|\cdot\|_{1}$.

Proof. Let $A=\bigoplus_{k=1}^{N} M_{m_{k}}(\mathbb{C})$. For any $a=\left(\left(a_{i j}^{(1)}\right), \ldots,\left(a_{i j}^{(N)}\right)\right) \in A$, we define

$$
\|a\|_{s}=\sum_{k=1}^{N} \sum_{i, j=1}^{m_{k}}\left|a_{i j}^{(k)}\right|
$$

Since $\|\cdot\|_{s}$ is equivalent to the $C^{*}$-norm $\|\cdot\|_{A}$, on $A$, there exists a $\kappa>0$ such that $\|a\|_{A} \leq \kappa\|a\|_{s}(a \in A)$. Therefore, if $f(t)=\left(\left(f(t)_{i j}^{(1)}\right), \ldots,\left(f(t)_{i j}^{(N)}\right)\right) \in A$, then

$$
\|f\|_{1} \leq \kappa \sum_{t \in G} \sum_{k=1}^{N} \sum_{i, j=1}^{m_{k}}\left|f(t)_{i j}^{(k)}\right| .
$$

Let $\pi^{(k)}$ be the representation defined by $\pi^{(k)}\left(a^{(1)}, \ldots, a^{(N)}\right)=\pi\left(0, \ldots, 0, a^{(k)}, 0\right.$, $\ldots, 0)$. There exists $\xi_{i} \in H$ such that $\left\|\xi_{i}\right\| \leq 1$ and $\left|f(t)_{i j}^{(k)}\right| \leq\left|\left\langle\pi^{(k)}(f(t)) \xi_{j}, \xi_{i}\right\rangle\right|$ $(t \in G)$. Thus, for fixed $i$ and $j$,

$$
\sum_{t \in G}\left|f(t)_{i j}^{(k)}\right| \leq \sum_{t \in G}\left\|\pi^{(k)}(f(t)) \xi_{j}\right\| \leq n_{\pi, 1}(f) .
$$

Consequently, $n_{\pi, 1}(f) \leq\|f\|_{1} \leq \kappa\left(\sum_{k=1}^{N} m_{k}^{2}\right) n_{\pi, 1}(f)$.

Example 3.4. Suppose that $G=\mathbb{Z}$ and $A=\mathcal{K}\left(l^{2}(\mathbb{Z})\right)$. Let $\left\{e_{k}\right\}_{k \in \mathbb{Z}}$ be the canonical basis for $l^{2}(\mathbb{Z})$ and let $p^{(k)} \in A$ be defined by $p^{(k)}\left(e_{l}\right)=\delta_{k, l} e_{k}$. Fix $m \in \mathbb{N}$. Define $f_{m} \in K(G ; A)$ by

$$
f_{m}(k)= \begin{cases}p^{(k)} & \text { if }|k| \leq m, \\ 0 & \text { otherwise. }\end{cases}
$$


Then clearly $\left\|f_{m}\right\|_{1}=2 m+1$. However, if $\pi$ is the canonical representation of $A$ on $l^{2}(\mathbb{Z})$, then

$$
\begin{aligned}
n_{\pi, 1}\left(f_{m}\right) & =\sup \left\{\sum_{k=-m}^{m}\left\|\pi\left(f_{m}(k)\right) \xi\right\|: \xi \in l^{2}(\mathbb{Z}) ;\|\xi\| \leq 1\right\} \\
& =\sup \left\{\sum_{k=-m}^{m}\left|\xi_{k}\right|:\left(\xi_{l}\right) \in l^{2}(\mathbb{Z}) ; \sum_{k \in \mathbb{Z}}\left|\xi_{l}\right|^{2} \leq 1\right\}=\sqrt{2 m+1} .
\end{aligned}
$$

Therefore, $n_{\pi, 1}$ is not equivalent to $\|\cdot\|_{1}$.

Proposition 3.5. Suppose that $(\pi, u, H)$ is a covariant representation of $(A, G, \alpha)$. Then $\|f\|_{\pi, 1}=\max \left\{n_{\pi, 1}(f), n_{\pi, 1}\left(f^{\#}\right)\right\}$ is an involutive algebra semi-norm on $(K(G, A), \star, \#)$. If $l_{\pi}^{1}(G ; A)$ is the completion of the quotient of $K(G ; A)$ under $\|\cdot\|_{\pi, 1}$, then there exists a contractive Banach *-algebra homomorphism $\epsilon_{\pi}$ from $l^{1}(G ; A)$ to $l_{\pi}^{1}(G ; A)$. Moreover, there exists a contractive representation $\mu_{\pi, u}$ of $l_{\pi}^{1}(G ; A)$ on $H$ such that $\mu_{\pi, u} \circ \epsilon_{\pi}=u \star \pi$.

Proof. For any $g \in K(G ; A)$ and $\xi \in H$, we have

$$
\begin{aligned}
\sum_{s \in G}\|\pi(f \star g(s)) \xi\| & \leq \sum_{r \in G} \sum_{t \in G}\left\|\pi\left(\alpha_{r^{-1}}(f(t))\right) \pi(g(r)) \xi\right\| \\
& =\sum_{r \in G} \sum_{t \in G}\left\|\pi(f(t)) u_{r} \pi(g(r)) \xi\right\| \\
& \leq \sum_{r \in G} n_{\pi, 1}(f)\left\|u_{r} \pi(g(r)) \xi\right\|=n_{\pi, 1}(f) n_{\pi, 1}(g)\|\xi\| .
\end{aligned}
$$

Thus, $n_{\pi, 1}$ is an algebra semi-norm on $(K(G ; A), \star)$ and $\|\cdot\|_{\pi, 1}$ is an involutive algebra semi-norm on $(K(G ; A), \star, \#)$. The second statement of the proposition follows from Remark $3.2\left(\right.$ a). Finally, as $\|u \star \pi(f)\| \leq n_{\pi, 1}(f)$, the third statement is easy to obtain.

Remark 3.6. (a) We can regard $l_{\pi}^{1}(G ; A)$ as the completion of the quotient of $l^{1}(G ; A)$ with respect to $\|\cdot\|_{\pi, 1}$. In this case, the completion of the quotient of $l^{1}(G ; A)^{\sim}$ with respect to $\|\cdot\|_{\pi, 1}$ coincides with $l_{\pi}^{1}(G ; A)^{\sim}$.

(b) Suppose that $\pi$ is faithful. Then any $x \in l_{\pi}^{1}(G ; A)$ defines a map $f: G \rightarrow A$ such that

$$
\sup \left\{\sum_{t \in G}\|\pi(f(t)) \xi\|: \xi \in H \text { with }\|\xi\| \leq 1\right\}<\infty
$$

(because any sequence in $K(G ; A)$ converging to $x$ will converge pointwisely and the pointwise limits of any two such sequences are the same). Thus, $\epsilon_{\pi}: l^{1}(G ; A) \rightarrow$ $l_{\pi}^{1}(G ; A)$ (Proposition 3.5) is injective. Moreover, $\epsilon_{\pi}$ extends to an injection from $l^{1}(G ; A)^{\sim}$ to $l_{\pi}^{1}(G ; A)^{\sim}$.

(c) Let $G=\mathbb{Z}$ and $A=\mathcal{K}\left(l^{2}\right)$, and let $\alpha$ be the trivial action. If $\pi$ is the canonical representation of $A$ and $u$ is the trivial representation of $G$ respectively on $l^{2}$, then $\left(\pi, u, l^{2}\right)$ is a covariant representation. Suppose that $\epsilon_{\pi}$ is surjective. Then the Open Mapping theorem will imply that $\|\cdot\|_{\pi, 1}$ is equivalent to $\|\cdot\|_{1}$, which contradicts the conclusion of Example 3.4 (note that $\|\cdot\|_{\pi, 1}=n_{\pi, 1}$ in this case). Therefore, $\epsilon_{\pi}$ is in general not surjective. 
Lemma 3.7. Suppose that $G$ is a polynomial growth discrete group and $(\pi, H)$ is a representation of $A$. If $f \in K(G ; A)$ and $f=f^{\#}$, then $\epsilon_{\pi}(f)$ has slow growth.

Proof. Let $u(f)$ be as in Lemma 2.2(a) and let $\mu: l^{1}(G ; A) \rightarrow A \times_{\alpha} G$ be the canonical $*$-homomorphism. Since $\mu(u(f))=u(\mu(f))$, we have

$$
\mu\left(u(f)^{\#} \star u(f)\right) \leq \mu(f \star f)
$$

(by an analogue of [3, Lemme 4] for $C^{*}$-algebras). If $E$ is the canonical conditional expectation from $A \times{ }_{\alpha} G$ to $A$ (see e.g. [4]), then $E(\mu(g))=g(e)$ for any $g \in$ $l^{1}(G ; A)$. Therefore, using (3.2),

$$
\begin{aligned}
\sum_{t \in G} u(f)(t)^{*} u(f)(t) & =\left(u(f)^{\#} \star u(f)\right)(e) \\
& =E\left(\mu\left(u(f)^{\#} \star u(f)\right)\right) \leq E(\mu(f \star f))=\sum_{t \in G} f(t)^{*} f(t) .
\end{aligned}
$$

Consequently, for any $\xi \in H$ with $\|\xi\| \leq 1$, we have

$$
\begin{aligned}
\sum_{t \in G}\|\pi(u(f)(t)) \xi\|^{2} & =\left\langle\pi\left(\sum_{t \in G} u(f)(t)^{*} u(f)(t)\right) \xi, \xi\right\rangle \leq\left\|\sum_{t \in G} u(f)(t)^{*} u(f)(t)\right\| \\
& \leq\left\|\sum_{t \in G} f(t)^{*} f(t)\right\| \leq n_{\pi, 2}(f)^{2}
\end{aligned}
$$

(see Remark 3.2(b)). Thus, $n_{\pi, 2}(u(f)) \leq n_{\pi, 2}(f)$. Let $S$ be the support of $f$. As $G$ has polynomial growth, there exists $N \in \mathbb{N}$ such that $\left|S^{m}\right|=O\left(m^{N}\right)$. Now, a similar argument to that of [3, Lemme 6] will give the result, but since we are in a slightly different setting, we will sketch the proof here for clarity. If $m \in \mathbb{N}$ and $\xi \in H$ with $\|\xi\| \leq 1$, then

$$
\begin{aligned}
\sum_{t \in S^{m^{2}-1}}\|\pi(u(m f)(t)) \xi\| & \leq\left(\sum_{t \in S^{m^{2}-1}}\|\pi(u(m f)(t)) \xi\|^{2}\right)^{1 / 2}\left|S^{m^{2}-1}\right|^{1 / 2} \\
& \leq n_{\pi, 2}(u(m f))\left|S^{m^{2}-1}\right|^{1 / 2} \leq m n_{\pi, 2}(f)\left|S^{m^{2}-1}\right|^{1 / 2} \\
& \leq C_{1} m^{N+1}
\end{aligned}
$$

$\left(C_{1}\right.$ is independent of $\xi$ and $\left.m\right)$. On the other hand, the same argument as that for [3. Lemme 6] shows that

$$
\begin{aligned}
\sum_{t \in G \backslash S^{m^{2}-1}}\|\pi(u(m f)(t)) \xi\| & \leq \sum_{t \in G \backslash S^{m^{2}-1}}\left\|\sum_{k=m^{2}}^{\infty} \frac{(i m f)^{k}}{k !}(t)\right\| \\
& \leq C_{2} m^{-m^{2}-1} e^{m^{2}+m}
\end{aligned}
$$

$\left(C_{2}\right.$ is independent of $\xi$ and $m$ ). Equations (3.3) and (3.4) imply that $n_{\pi, 1}(u(m f))=$ $O\left(m^{N+1}\right)$. Finally, let $[\lambda]$ be the integral part of $\lambda$. Then we have

$$
n_{\pi, 1}\left(e^{i \lambda f}\right)=n_{\pi, 1}\left(e^{i(\lambda-[\lambda]) f} e^{i[\lambda] f}\right) \leq e^{\|f\|_{1}}\left(1+n_{\pi, 1}(u(i[\lambda] f))\right)=O\left(|\lambda|^{N+1}\right) .
$$

Again, using the argument of [3, Lemme 7] and Lemma 1.4(a), we have the following theorem. 
Theorem 3.8. Suppose that $G$ is a discrete polynomial growth group. Let $(A, G, \alpha)$ be a $C^{*}$-dynamical system and let $(\pi, u)$ be a covariant representation of $(A, G, \alpha)$. Let $f \in K(G ; A)$ with $f=f^{\#}$ and let $\varphi$ be a smooth and integrable complex function on $\mathbb{R}$.

(a) The Bochner integral $\varphi_{\pi}\{f\}:=\frac{1}{2 \pi} \int_{-\infty}^{\infty} \hat{\varphi}(\lambda) \epsilon_{\pi}\left(e^{i \lambda f}\right) d \lambda$ exists in $l_{\pi}^{1}(G ; A)^{\sim}$ and $\varphi_{\pi}\{f\} \in l_{\pi}^{1}(G ; A)$ if $\varphi(0)=0$.

(b) Suppose that $u \star \pi$ extends to a faithful representation of $A \times{ }_{\alpha} G$. Then one can regard $\mu_{\pi, u}$ as a $*$-homomorphism from $l_{\pi}^{1}(G ; A)^{\sim}$ to $A \times_{\alpha} G$. Under this identification, for any covariant representation $(\nu, v)$ of $(A, G, \alpha)$, we have $(v \star \nu)\left(\mu_{\pi, u}\left(\varphi_{\pi}\{f\}\right)\right)=\varphi((v \star \nu)(f))$.

(c) Suppose that $u \star \pi$ is faithful on $A \times_{\alpha} G$. Then $l_{\pi}^{1}(G ; A)$ is $*$-regular.

Remark 3.9. (a) Suppose that $u \star \pi$ extends to a faithful representation of $A \times{ }_{\alpha} G$. By Theorem 3.8(b), any non-degenerate $*$-representation of $A \times{ }_{\alpha} G$ induces (through the map $\left.\mu_{\pi, u}\right)$ a non-degenerate $*$-representation of $l_{\pi}^{1}(G ; A)$. On the other hand, any non-degenerate $*$-representation of $l_{\pi}^{1}(G ; A)$ induces (through the map $\epsilon_{\pi}$ in Proposition 3.5) a non-degenerate *-representation of $l^{1}(G ; A)$. Since $\mu_{\pi, u} \circ \epsilon_{\pi}$ is the canonical embedding of $l^{1}(G ; A)$ in $A \times_{\alpha} G$, we see that the enveloping $C^{*}$ algebra of $l_{\pi}^{1}(G ; A)$ is again $A \times{ }_{\alpha} G$.

(b) By Proposition 3.3, Theorem 3.8 can be regarded as a partial generalization of Theorem 2.4.

\section{REFERENCES}

1. M. Baillet, Analyse spectrale des opérateurs hermitiens d'une espace de Banach, J. London Math. Soc. (2) 19 (1979), 497-508. MR0540066 (80j:46080)

2. J. Boidol, H. Leptin, J. Schürman and D. Vahle, Räume primitiver Ideale von Gruppenalgebren, Math. Ann. 236 (1978), 1-13. MR0498971 (58:16959)

3. J. Dixmier, Opérateurs de rang fini dans les représentations unitaires, Inst. Hautes Études Sci. Publ. Math. No. 6 (1960), 13-25. MR0136684 (25:149)

4. R. Exel, Amenability for Fell bundles, J. Reine Angew. Math. 492 (1997), 41-73. MR.1488064 (99a:46131)

5. U. Haagerup, On the dual weights for crossed products of von Neumann algebras I-Removing separability conditions, Math. Scand. 43 (1978/79), 99-118. MR0523830 (81e:46048a)

6. A. Kishimoto, Ideals of $C^{*}$-crossed products by locally compact abelian groups, Proc. Symp. Pure Math. 38 (1982), 365-368. MR0679718 (84b:46086)

7. T. W. Palmer, Banach algebras and the general theory of $*$-algebras. Vol. 2, Encyclopedia of Mathematics and its Applications 79, Camb. Univ. Press (2001). MR 1819503 (2002e:46002)

Department of Mathematics, The Chinese University of Hong Kong, Hong Kong

E-mail address: cwleung@math.cuhk.edu.hk

School of Mathematical Sciences and LPMC, Nankai University, Tianjin 300071, PeoPle's Republic of China

E-mail address: ckng@nankai.edu.cn 